\title{
L'islam des musulmans de Tanna (Vanuatu)
}

\author{
Marc Tabani
}

La PRÉSEnCE de l'islam dans certaines îles du Pacifique Sud a parfois précédé celle du christianisme. Les peuples mélanésiens occupant les marges occidentales de la Nouvelle-Guinée ont été en contact avec des marchands arabes, depuis au moins le $\mathrm{Xv}^{\mathrm{e}}$ siècle, puis en relation, à partir du XvII ${ }^{\mathrm{e}}$ siècle, avec des sultanats établis dans le nord des Moluques (Slama 2015: 249). À Fidji et en Nouvelle-Calédonie, l'installation de musulmans est historiquement liée à l'arrivée de populations étrangères (prisonniers condamnés au bagne ou travailleurs sous contrat), à partir du milieu du xix ${ }^{\mathrm{e}}$ siècle (Ali 2004; Kohler 1982). Avec son incorporation à l'Indonésie en 1962, la Papouasie occidentale, ex-colonie néerlandaise, est devenue une province de l'État qui compte le plus grand nombre de musulmans au monde. Toutefois, en dehors de ces quelques exceptions, l'islam a été, jusqu'à récemment, peu présent dans la région du Pacifique, «statistiquement la plus chrétienne au monde» (Robbins 2007: 33).

Les recherches effectuées par Debra McDougall (2009) aux îles Salomon et par Scott Flower (2017) en Papouasie-Nouvelle-Guinée (PNG) apportent, au sujet du développement de l'islam en Mélanésie, de précieuses contributions ethnographiques et d'utiles points de comparaison. Les conditions sociopolitiques et les effets culturels de son apparition dans ces deux archipels présentent de nombreuses caractéristiques communes, que l'on retrouve également à Vanuatu. La multiplication des conversions à cette religion procède, dans ces trois pays, d'une dynamique initiée au cours de leur histoire d'États postcoloniaux.

Dans cette partie de la Mélanésie, des populations autochtones ont adopté l'islam après l'avoir elles-mêmes importé. Même si les données statistiques concernant le nombre de musulmans demeurent lacunaires, 
la progression des fidèles de cette religion minoritaire est néanmoins bien attestée. En témoignent les estimations fondées sur de rares statistiques complétées par des données de terrains recueillies par Scott Flower (2017: 17-18), au cours des années 2010: entre 4500 et 5000 fidèles en PNG (sur une population totale de plus de 8200000 habitants), 2000 aux Salomon (pour plus de 610000 habitants) et 500 à Vanuatu (pour plus de 270000 habitants) ${ }^{1}$. D'après le dernier recensement national ${ }^{2}$, à Vanuatu, près de $70 \%$ de la population a déclaré une affiliation religieuse chrétienne. En quelques décennies, l'islam a conforté sa pérennité dans cette région, comptant désormais trois générations de fidèles par l'intermédiare desquelles de nombreux liens avec des coreligionnaires et des organisations islamiques à l'étranger ont été tissés. De cette implantation résultent des conséquences similaires quant à l'évolution des paysages religieux dans ces trois États insulaires, depuis leur accession respective à l'indépendance (de 1975 à 1980). L'islam joue un rôle actif dans la recomposition des formes institutionnelles préexistantes de pluralisme religieux.

Debra McDougall et Scott Flower ont également souligné que l'adhésion à l'islam est marquée par une certaine continuité. La seule rupture apparente avec le christianisme serait d'ordre idéologique, sans aucune incidence majeure sur le mode de vie des fidèles. Selon McDougall, les musulmans des Salomon «abordent leur nouvelle foi avec le regard de celle qu'ils souhaitent quitter» (2009: 483) ${ }^{3}$. De même, en PNG, les nouveaux convertis jugent fréquemment l'islam plus apte que le christianisme à assurer la permanence des traditions (Flower 2015: 59). Toutefois, qu'elle soit affirmée ou contestée, cette continuité n'en demeure pas moins problématique lorsque l'implantation de l'islam s'avère conflictuelle, comme c'est actuellement le cas dans cette partie de la Mélanésie, où les discours polémiques sur la rigidité supposée de l'islam à l'égard du christianisme ou des cultures locales se multiplient. Le danger d'un abandon progressif du christianisme, agité par des leaders religieux ou politiques chrétiens, se voit placé sur le même plan que celui d'une islamisation des cultures mélanésiennes. Chrétiens et musulmans s'accusent réciproquement de vouloir imposer leur vision de la coutume à des fins politiques. Certaines critiques qui nourrissent ces rivalités ne sont pas sans rappeler les oppositions exacerbées, à l'époque coloniale, dans des îles comme Tanna (sud de Vanuatu), entre partisans et détracteurs de mouvements millénaristes, connus alors sous l'appellation dépréciative de "cultes du cargo».

1. Nombre des habitants par pays selon les indicateurs de la Banque mondiale.

2. Cf. Vanuatu National Statistic Office (VNso 2009: 76-77).

3. Toutes les citations extraites d'ouvrages ou d'articles en anglais ont été traduites par mes soins. Quant aux entretiens, ils ont été menés principalement en bislama. 
Lobjectif de cet article est d'exposer les spécificités de l'islam à Vanuatu, la pratique de cette religion étant encore très peu documentée dans cet archipel. À partir de mes observations et données ethnographiques ${ }^{4}$, j'examinerai les implications sociales et institutionnelles liées à son implantation dans plusieurs îles. Prétexte à un intense déploiement de stratégies idéologiques antagonistes, l'islam soulève à Vanuatu des enjeux politiques et identitaires, tant à une l'échelle nationale que locale. Dans ce cadre, j'accorderai une attention particulière aux représentations et aux discours formulés sur la continuité ou, à l'inverse, sur les transformations induites par ce mouvement de conversion comparé aux vagues de christianisation qui l'ont précédé et aux syncrétismes qu'elles ont contribué à diffuser. Envisager l'émergence inédite de cette pratique religieuse à Vanuatu en tant que quête d'une modernité non occidentale alternative, destinée à renforcer les traditions, m'amènera à proposer quelques éléments de réflexion sur la problématique des modes de compatibilité entre religions importées et cultures mélanésiennes contemporaines.

Dans les premières sections de cet article, j'analyserai comment un sentiment d'hostilité prend racine à Vanuatu, par assimilation d'un discours critique envers l'islam importé de l'étranger. Relayé dans les médias et les réseaux sociaux, celui-ci insiste notamment sur les incompatibilités supposées de l'islam avec deux des points affirmés dans le préambule de la Constitution, à savoir la primauté accordée aux valeurs mélanésiennes et aux principes chrétiens. Ainsi, par exemple, l'importance du cochon dans les échanges sociaux, la consommation du $k a v a^{5}$ et diverses autres pratiques quotidiennes (coutumes vestimentaires, habitudes alimentaires), cérémonielles (rites et fêtes d'origine païenne) ou politiques (chefs coutumiers), tous ces us et coutumes amalgamés dans la notion de kastom $^{6}$, sont des

4. Mon intérêt pour la question de l'islam à Vanuatu date de février 2008, lors de mes premiers échanges avec le chef Jimmy Noankan, le plus ancien musulman de Tanna, un personnage sur lequel nous reviendrons plus loin. Mes données éparses sur le sujet ont été consolidées par des enquêtes ethnographiques menées à Port-Vila et à Tanna, d'octobre à décembre 2017, doublées d'une veille documentaire sur le sujet.

5. Breuvage traditionnel intoxicant obtenu à partir des racines de la plante du même nom. La consommation du cochon et du kava est également prohibée par certaines Églises chrétiennes, notamment par les adventistes.

6. La notion de kastom (en bislama, la langue nationale) couvre un champ sémantique plus large que celle, anglaise, de custom dont elle tire son étymologie. Lorsqu'elle est utilisée pour désigner des réalités locales, elle s'applique à tout élément culturel perçu comme traditionnel, sans être nécessairement autochtone ni forcément ancien, dont l'historicité demeure vague bien que présentée comme incontestable. L'usage politique de ce concept entraîna sa transcription dans la Constitution avec la formule "valeurs mélanésiennes ", élevant son intérêt symbolique à celui d'un fondement culturel dans lequel le processus d'édification nationale devait être ancré. Depuis lors, la kastom englobe, pour reprendre la formule de Ton Otto et Poul Pedersen (2005), à la fois des «traditions traditionnelles» et des «traditions traditionalistes». La problématique des instrumentalisations idéologiques de la kastom en Mélanésie, mise en évidence par Roger Keesing et Robert Tonkinson dans un numéro précurseur de la revue Mankind (1982), a donné lieu à un vaste et long débat, 
marqueurs identitaires régulièrement évoqués dans les débats publics. À propos de l'islam, ces symboles sont mobilisés pour démontrer l'altérité supposément radicale des pratiques musulmanes, «importées d'Arabie», et inciter à leur rejet.

Les sections suivantes seront consacrées aux représentations et aux discours des fidèles venant contredire l'incompatibilité de l'islam avec les traditions religieuses et culturelles déjà présentes sur place. Nous verrons ainsi que, dans l'île de Tanna, au sud de Vanuatu, la conversion à cette religion est interprétée et vécue comme une revitalisation des pratiques ancestrales, cette vision se trouvant facilitée par le tropisme millénariste lié au mouvement John Frum que la littérature anthropologique sur les «cultes du cargo" en Mélanésie a contribué à faire connaître (Lindstrom 1993; Tabani 2008). Au-delà des raisons économiques et politiques ayant favorisé leur conversion, les musulmans de Tanna affirment l'existence d'une véritable osmose culturelle et spirituelle de l'islam avec les pratiques coutumières (kastom $)^{7}$. Pour eux, l'islam n'est pas seulement une religion adaptable aux sociétés traditionnelles, mais se trouve intégré, tout comme la révélation chrétienne qui l'a précédé, à une cosmogénèse qui, en élaborant des mythologies syncrétiques, situe Tanna au centre et au commencement du monde. Si l'adoption de l'islam ne peut s'expliquer à Vanuatu sans l'expérience préalable de la christianisation, elle ne peut l'être à Tanna sans la référence au mouvement John Frum. Mais, avant d'en venir à ce cas insulaire spécifique, rappelons brièvement la configuration de l'échiquier religieux national.

\section{Les Églises et l'État à Vanuatu}

Les Églises aujourd'hui considérées comme «anciennes» (olfala joj, en bislama) ou historiques sont celles qui s'installèrent dans l'archipel avant ou peu après la création, en 1906, du Condominium franco-britannique des Nouvelles-Hébrides, puis durant l'entre-deux-guerres. Au cours de la période coloniale, les principales missions protestantes (presbytériennes dans l'ensemble des îles du sud de l'archipel, anglicanes au nord, avec quelques

[Suite de la note 6] souvent polémique, et à une abondante littérature sur le sujet (pour une compilation des nombreux écrits qui ont alimenté divers jalons de cette controverse, on pourra se reporter aux chapitres II et III de la thèse de Caroline Graille [2015]).

7. Si le choix d'adhérer à des Églises pentecôtistes, depuis leur essor à Tanna dans les années 1990, tend à se faire sur une base plus individuelle, il en va différemment dans le cas des bahaïs et des musulmans dont la conversion concerne principalement des familles élargies ou sous-groupes «tribaux", comme ce fut le cas, autrefois, pour les Églises historiques. Il en ressort que les justifications données reproduisent dans leurs grandes lignes les arguments développés par les représentants de ces groupes, en particulier le patriarche Jimmy Noankan et l'imam Cheikh Mustapha, d'où l'importance accordée à leurs propos. C'est à cette expression collective que je me réfere dans le cadre de cet article, lorsque j'évoque les sentiments ou les opinions des «musulmans de Tanna». 
implantations adventistes, apostoliques et de l'Église du Christ disséminées dans les principales îles) s'opposèrent régulièrement à l'Église catholique, notamment en raison du soutien qu'elles accordaient à leurs autorités de tutelle respectives, la Grande-Bretagne pour les premières, et la France pour la seconde. La nature de cet alignement était à la fois politique, économique et culturelle (Hassall 1991). L'héritage chrétien demeure une composante structurante de la diversité des identités culturelles contemporaines (Tabani 2002; Wittersheim 2006) ${ }^{8}$. Les distinctions confessionnelles contribuent encore de nos jours à entretenir des antagonismes culturels et idéologiques hérités de rivalités anciennes entre groupes (Miles 1998) et à maintenir une porosité des relations institutionnelles entre les Églises historiques et l'État.

La création d'un Conseil chrétien des Nouvelles-Hébrides (New Hebrides Christian Council, NHCC) remonte à 1967, dans un contexte marqué par le mouvement régional d'émancipation des Églises locales de leurs tutelles étrangères. Fondé à l'initiative de l'Église presbytérienne alors majoritaire, ce conseil était composé de représentants anglicans, catholiques, presbytériens, de membres de l'Église du Christ et de l'Église apostolique. Son but officiel était de travailler à une unité œcuménique, de surmonter les divisions confessionnelles, culturelles et linguistiques en vue de leur future indépendance. Mais, en contrepartie, les artisans de cette unité dans la pluralité s'accordèrent unanimement pour éliminer toute concurrence de nouvelles Églises ou sectes venues de l'étranger (Myers 1984: 346). Le Conseil chrétien de Vanuatu (Vanuatu Christian Council, Vcc) qui succéda au NHCC à l'indépendance en 1980, intégra l'Église pentecôtiste de l'Assemblée de Dieu et les adventistes, tout en continuant de réclamer la fermeture du pays à l'implantation de nouvelles congrégations. Gagnant en influence durant cette période, le VCC favorisa l'inscription des principes chrétiens dans la Constitution de la jeune république et accepta certains compromis quant à la reconnaissance de valeurs mélanésiennes et d'une kastom dépouillée de ses oripeaux païens. Les réseaux politiques de ses représentants contribuèrent également à faire de cette institution un acteur économique, désormais bénéficiaire de programmes d'aides et de développement internationaux. Lexclusivité fut accordée au VCC, auprès du gouvernement, en matière de conseil dans les affaires religieuses.

8. Le mot relijin, en bislama, est un anglicisme d'usage récent, absent par exemple du dictionnaire de Terry Crowley (1995). À l'époque coloniale étaient considérés comme "chrétiens» à Vanuatu, les groupes ayant quitté la coutume (kastom) pour se rattacher à une Église (skul). Selon que l'on appartenait à une Église réformée ou catholique, l'instruction religieuse se faisait en anglais ou en français. L'église et l'école étaient alors synonymes. Pour indiquer une appartenance religieuse non chrétienne, la formule consacrée est mi stap long kastom nomo («je suis seulement la coutume»). Les croyances coutumières locales ont été répertoriées dans les désignations officielles du recensement comme des «customary beliefs» et concerneraient 8 \% de la population (VNso 2009: 77). 
Les Églises restées en dehors du VCC ne purent guère faire entendre leur voix dans le jeu politique national. Les mormons et les témoins de Jéhovah, considérés comme "non chrétiens", furent laissés à l'écart, tout comme les bahaïs présents à Port-Vila et à Tanna depuis les années 1960 (Hassall 2005). Le VCC arriva ponctuellement à faire interdire des représentants de nouvelles Églises (dont la secte Moon, en 2005). Mais, à partir des années 1990, il perdit de son influence sur la société et les institutions, au fur et à mesure de la diminution régulière des membres des Églises qu'il regroupait et de l'augmentation des Églises néo-évangéliques. Le renouveau charismatique que connurent les Églises historiques à Vanuatu dans les années 1970-1980 déboucha dans les années 1990, sur une multiplication de groupes évangéliques dissidents et sur l'implantation de nombreuses Églises pentecôtistes étrangères. Franco Zocca dénombrait, dans les années 2000, plus de soixante affiliations religieuses revendiquées par des groupes sociaux de tout l'archipel, pour moins d'une dizaine dans les années 1970 (2006: 229, 232). Dix ans plus tard, à Port-Vila, Annelin Eriksen et Rose Andrew (2010) en recensent une cinquantaine, rien que parmi les chrétiens?

Quatre décennies après l'indépendance, l'opposition du VCC aux dissidences confessionnelles internes aux églises évangéliques et à l'importation de nouvelles congrégations se solde donc par un échec. Le pasteur presbytérien Alan Nafuki, président du VcC depuis 2016, est forcé de l'admettre. Il reconnait désormais «les diverses toutes petites fois, celles notamment des bahaïs, des mormons, des Jéhovah, car elles étaient déjà présentes avant l'indépendance» (Entretien, Port-Vila, 7 décembre 2017). Son hostilité demeure intacte à l'égard d'autres communautés religieuses, notamment contre les groupes pentecôtistes qui ont fait perdre à l'Église presbytérienne des milliers de ses fidèles. Il plaide pour des règles plus contraignantes que celles qui, jusqu’à présent, n’ont pas réussi à contenir la prolifération de nouvelles Églises. Mais, d'après lui, de nouvelles craintes se sont manifestées ces dernières années, avec l'apparition de l'islam, contre lequel il enjoint de lutter en priorité:

«Concernant l'islam, il s'agit d'une foi totalement différente, qui n'appartient qu'aux musulmans. Pour la diffuser, ils utilisent des fleurs, mais ces fleurs cachent un couteau tranchant. En Iran par exemple, une fois que la Bible fut traduite dans la langue de l'islam, de nombreux musulmans se sont convertis au christianisme, car ils pouvaient dès lors vérifier combien les discours de l'islam étaient mensongers. À cause de ça, ils ont été réprimés. C'est pourquoi nous ne voulons pas de l'islam à Vanuatu. L'islam

9. Selon Annelin Eriksen, la stratégie des Églises pentecôtistes consiste à reprendre une protestation sociale qui juge l'État et les anciennes Églises incapables de remédier aux maux dont souffrent les habitants du pays. Ces Églises tendent à se substituer à l'État et ambitionnent de «soigner la nation" dans son ensemble (2009: 70). 
n'est pas comme la foi bahaïe, c'est une grande et puissante religion. Les gens qui ont rejoint l'islam sont venus me voir pour me dire que c'était une bonne religion et qu'ils aideraient à promouvoir le Vanuatu Christian Council. Mais ce n'est là qu'un des visages de l'islam, un autre est de vouloir s'emparer du pouvoir. Étant donné que la liberté de croyance est inscrite dans notre Constitution, nous avons décidé, avec les parlementaires, qu'il fallait la réviser et ajouter, comme conditions à la venue de nouvelles religions, l'obligation pour elles de dire la vérité et de montrer du respect. Si par exemple quelqu'un veut se convertir à l'islam, il se doit d'avertir les membres de sa communauté et son chef. Le chef pourra alors constater que cette personne veut en fait établir une nouvelle Église, et il devra se poser la question: "Est-ce une Église qui veut unir ou diviser?". Nous soutiendrons alors l'avis du chef s'il s'aperçoit d'une absence de respect [...]. Nous avons prévenu le gouvernement pour qu'il refuse [la construction de toute nouvelle mosquée], et attende la proclamation de la "loi du respect". À Tanna, les autorités provinciales n'ont pas pu empêcher d'en construire une dans un village [à Iwel, dans la région du Centre Brousse], car les musulmans y sont nombreux. C'est d'ailleurs parce qu'ils ont à Tanna des gens qui vivent intégralement dans la coutume ou adhèrent à des mouvements comme John Frum que nous ne pouvons pas simplement changer la Constitution [...]. De nouvelles religions comme l'islam peuvent faire une demande pour s'installer, et c'est au Vanuatu Christian Council sous l'ordre du gouvernement d'y répondre. La liberté religieuse n'a plus cours comme autrefois, il y a une condition: le respect ${ }^{10}$ " (Entretien, Port-Vila, 7 décembre 2017).

À l'appel de Nafuki, une première manifestation a été organisée par l'Église presbytérienne, en octobre 2016, mobilisant deux à trois cents de ses membres dans les rues de Port-Vila pour réaffirmer les fondements chrétiens du pays, tout en y dénonçant la progression de l'islam ${ }^{11}$. Le pasteur presbytérien Tallis Obed Moses, élu à la présidence de la République en juillet 2017, se déclarait en faveur d'une révision constitutionnelle allant dans le sens de la restriction de la liberté de culte pour les religions non chrétiennes ${ }^{12}$, et affirmait que "la seule religion qui existe à Vanuatu est le christianisme» ${ }^{13}$. Cette surenchère dans les mesures drastiques à prendre contre l'islam contraste avec l'indifférence témoignée antérieurement, pendant des années, aux musulmans par les autorités politiques et religieuses du pays.

La présentation de quelques éléments historiques et sociologiques sur les musulmans de Vanuatu permettra de retracer la genèse du discours anti-islamique.

10. Lamont Lindstrom a analysé, au sein de la société de Port-Vila, l'importance accordée au respek. S'il valorise la bonne conduite dans les rapports sociaux, ce «mot-valise» prend un sens hiérarchique marqué, sans référence particulière à des valeurs d'égalité individuelle (2017: 25).

11. Cf. Lene Garae, "Vcc Proclams Vanuatu for God», Vanuatu Daily Post, 22 octobre 2016.

12. Cf. Lene Garae, "President Urges Constitutional Amendment», Vanuatu Daily Post, 6 octobre 2017.

13. Cf. Lene Garae, "President: "Vanuatu Has One Religion, Christianity" ", Vanuatu Daily Post, 10 octobre 2017. 


\section{Les premiers musulmans de Mele}

Pendant plus d'une décennie, Henry Hussein Nabanga fut le seul musulman ni-Vanuatu ( citoyen de Vanuatu»). Sa biographie est évoquée dans une brochure prosélytiste sur la naissance de l'islam dans cet archipel (Ahmadu \& Shuaibu 2004: 22-23). En 1973, à l'âge de 30 ans, il partit en Inde pour suivre une formation religieuse en traduction biblique. Il y découvrit l'islam et se convertit en 1978, à son retour au pays. À Mele, village périurbain de Port-Vila, son prosélytisme sut convaincre quelques membres de son groupe familial. Jusqu'à sa mort en 1993, sa prédication entrâna une centaine de conversions, la plupart des convertis étant d'anciens presbytériens passés ensuite par l'Église du Neil Thomas Ministry. Une salle de prière fut installée à Mele en 1992 et la Société islamique de Vanuatu (Vuanutu Islamic Society, VIs) fut enregistrée en 1997 auprès de la commission des services financiers, en tant qu'association caritative. Mustapha Kalaos, puis Mohammed Seddiq Sambo en furent les présidents. Des liens familiaux avec Nabanga, le premier converti du pays, ont longtemps permis aux musulmans de Mele d'occuper une place privilégiée dans la représentation officielle des communautés musulmanes du pays.

Les qualités personnelles et la morale irréprochable de Nabanga sont mises en avant par les responsables actuels de la VIs, pour expliquer le succès de l'enseignement de ce précurseur. D'après Mustapha Kalaos, «Il apprenait l'islam d'une manière qui prenait socialement tout son sens, [...] qu'il était un mode de vie complet, dans ce monde comme dans le suivant». La prédication de Nabanga fut d'autant mieux entendue, toujours selon Kalaos, que l'islam reconnaît le même Dieu. Le respect des musulmans pour les autres religions et leur proximité avec les chrétiens plaident en faveur d'une réunification autour d'une foi originelle, «la religion d'Adam": «ma coutume, ma culture [...] sont très similaires à la vie dans l'islam». Les règles censées être partagées n'en demeurent pas moins abstraites: "le respect, l'honnêteté, la responsabilité, l'impartialité, la justice, les pratiques alimentaires et des modes de vie au sein de la communauté» ${ }^{14}$.

L'engagement ultérieur du professeur de droit Mohammed Lawal Ahmadu fut tout aussi déterminant pour l'enracinement de l'islam au-delà de son ancrage urbain, et pour son extension en milieu rural dans plusieurs îles de l'archipel. Ce ressortissant nigérian vécut à Vanuatu de 1995 à 2007. Venu poursuivre ses études de droit à l'Université du Pacifique Sud (University of South Pacific, Suva, îles Fidji), avant d'y enseigner, il découvrit à PortVila un islam embryonnaire, ne dépassant pas les frontières de la capitale.

14. Cf. Shirley Joy, "Muslims in Vanuatu», Vanuatu Daily Post, 20 octobre 2004. 
Son prosélytisme s'accompagnait de la recherche d'aides en faveur de ses coreligionnaires ni-Vanuatu, et favorisa l'ouverture de leur communauté vers le monde islamique extérieur. Des accords furent d'abord passés avec l'Université de Kuala Lumpur pour l'accueil d'étudiants, puis avec le Conseil régional islamique Da'wah de l'Asie du Sud-Est et du Pacifique (Regional islamic Da'wah Council of South East Asia and the Pacific) pour le financement de bourses. Seddiq Sambo pouvait déclarer en 2007: «En ce moment même, vingt-huit musulmans de Vanuatu étudient dans des écoles islamiques outre-mer: à Fidji, en Malaisie, en Nouvelle-Zélande, en Arabie Saoudite, en Iran et au Pakistan ${ }^{15}$. De fil en aiguille, des liens officiels avec d'importantes organisations islamiques internationales ont été établis, dont l'Association mondiale de la jeunesse musulmane en Australie et Pacifique Sud (World Association for Muslim Youth Australia \& South Pacific), l'Aide aux musulmans d'Australie (Muslim Aid Australia) et la Banque islamique de développement (Islamic Development Bank, IDB). L'IDB, en plus de bourses et de séjours d'études, a financé, dans le quartier de Bladinières, le terrain et les bâtiments du Centre d'études islamiques de Port-Vila, qui sert également de salle de prière et assure le paiement des salaires des deux imams du pays. La construction d'une grande mosquée, dans un style architectural islamique, serait également en projet ${ }^{16}$.

Mohammed Lawal Ahmadu réussit également à convaincre des hommes influents, tel le chef Noankan de Tanna qui fut à l'origine d'une vague de conversion dans son île:

«L'islam à Tanna débuta en 1999. En tant que responsable politique, j'effectuai un séjour à Port-Vila et, à cette occasion, j'ai rencontré dans la rue un professeur de droit de l'Université Sud Pacifique, Mohamed, un Africain. Je pus constater au cours de notre discussion combien il respectait la kastom des ni-Vanuatu et combien la kastom de Tanna est semblable à celle de la religion muslam. J'ai alors souhaité me convertir, ce qui fut fait le 24 décembre, alors que Mohamed passait quatre jours chez moi, à Tanna. Il m’a aussi déclaré qu'il pouvait aider les enfants des pauvres pour l'école. Il a obtenu des bourses. J'ai pu ainsi envoyer des jeunes de mon groupe faire des études en Malaisie, à Fidji, en Indonésie, au Pakistan, en Afrique» (Entretien, Iwel, 27 novembre 2017).

D’après les imams Abdul Karim et Cheikh Mustapha ${ }^{17}$, le nombre de musulmans à Vanuatu ne dépasserait pas 500 personnes, dont un peu plus

15. Cité par Ben Bohane, dans son article «Green Moon Rising: Islam Is Spreading In Melanesia», Pacific Magazine, 29 juin 2007.

16. Entretiens avec Abdul Karim (Port-Vila, $1^{\text {er }}$ décembre 2017) et avec Cheikh Mustapha (Iwel, 17 novembre 2017). Alors âgés de 30 et 34 ans, ces deux petits-fils en ligne paternelle de Jimmy Noankan sont les premiers ni-Vanuatu et man Tanna à avoir été formés pour être imams pendant plusieurs années dans des écoles coraniques (madrasas) à Fidji (en 2000 à Lambasa, pour le premier, et en 2004 à Suva, pour le second).

17. Entretiens de novembre et décembre 2017, cf. supra note 16. 
d'une cinquantaine sont des résidents étrangers. Port-Vila et ses zones périurbaines compteraient 150 convertis originaires d'Éfaté, une centaine de ni-Vanuatu d'autres îles (dont une moitié de Tanna), une cinquantaine d'Indo-Fidjiens, quelques Salomonais et autres résidents étrangers. Un peu plus d'une centaine de musulmans vivent sur l'île de Tanna, et quelques dizaines d'autres, des familles ou individus, sont dispersés dans l'archipel. Ces estimations sont cinq fois supérieures à celles suggérées par Franco Zocca pour 2004 (2006: 259). Sambo, alors président de la VIs, a avancé en 2016, au cours d'une interview dans le Vanuatu Daily Post, le nombre de 1000 ni-Vanuatu musulmans, un chiffre délibérément exagéré, destiné à marquer les esprits, et repris également par le $\mathrm{VCC}^{18}$. Il insiste sur une progression de l'islam, qui ferait des musulmans une force politique, et annonce son intention de présenter la candidature d'un membre de sa communauté au parlement. Les étudiants régulièrement envoyés dans des pays islamiques pour y être formés seraient le gage d'une réussite économique des ni-Vanuatu musulmans profitant à tous les autres citoyens. Lui-même, en revanche, n'aurait bénéficié d'aucun enrichissement matériel. Par cette justification, il ne répondrait pas seulement aux critiques du camp adverse, mais également aux griefs de certains musulmans, comme ceux du chef Noankan:

«L'islam est d'abord arrivé à Mele, mais cela n'a pas fonctionné. Il y a eu des actions, des aides financières de l'étranger, pour des projets, pour aider financièrement les pauvres à Vanuatu, mais ce sont les gens de Mele qui ont usé de cet argent. À Mele on prie surtout pour obtenir des choses. Quand ils obtiennent quelque chose, ils le gardent pour eux. Aujourd'hui, ils sont devenus jaloux des man Tanna [insulaires de Tanna]. Après m'être converti, c'est moi qui me suis rendu à Vila, qui ai agi à Mele, prié et rassemblé des hommes pour qu'ils reviennent à l'islam; c'est moi qui ai porté le message d'Allah à Tanna. Désormais, mon île compte mille man muslam. Ils ont rejoint Allah et acceptent la kalima ["profession de foi"] » (Entretien, Iwel, 27 novembre 2017).

Les fidèles résidant à Port-Vila assistent à la prière du vendredi dans l'une des deux salles de prières situées à Mele ou à Bladinières. La première, d'une trentaine de mètres carrés, est rudimentaire, construite en parpaings et en tôles. En dehors des périodes de fêtes islamiques ou des visites de représentants d'organisations étrangères, la prière du vendredi n'y accueille qu'une dizaine de fidèles, selon ces derniers (seulement cinq lors de ma présence). Mais, depuis 2016, le centre de la vie religieuse s'est déplacé, dans le quartier de Bladinières. La VIs y dispose en effet d'un grand bâtiment bien équipé sur deux étages, comprenant des salles d'enseignement, des bureaux, une salle de prière pour les hommes, séparée de celle des femmes, et un logement de fonction pour l'imam. Les prières du vendredi, auxquelles j'ai assisté à

18. Cf. Lene Garae, "Over 1,000 Muslims», Vanuatu Daily Post, 27 octobre 2016. 
trois reprises, étaient dirigées par l'imam Abdul Karim. Aucun prêche ni sermon ne les accompagnaient. L'assistance y était cosmopolite et masculine, à l'exception des deux ou trois femmes aperçues qui étaient indo-fidjiennes. Les hommes étaient pour moitié étrangers, des résidents indo-fidjiens, quelques Pakistanais travaillant pour l'opérateur téléphonique Digicel et quelques étudiants salomonais. Les pratiquants ni-Vanuatu étaient tout autant originaires d'Éfaté et de diverses îles que de Tanna.

La chute de la fréquentation de la mosquée de Mele au profit de celle de Bladinières semble résulter d'une crise de croissance de la communauté musulmane, d'une diversification de ses composantes sociologiques et des profils individuels des musulmans, à Port-Vila. Les fidèles originaires d'Éfaté avec lesquels je me suis entretenu résident dans les zones périurbaines de Port-Vila (Mele, Pango, Erakor). Ils vivent sur leurs terres ou sur celles de leur groupe familial, et occupent des emplois de petits entrepreneurs, d'artisans, d'employés, ou de fonctionnaires. Bien intégrés dans la société, ils ont adopté un mode de vie urbain éloigné de la coutume, de sorte qu'ils n'accordent qu'une importance secondaire à la question d'éventuelles incompatibilités culturelles avec l'islam. En général, les musulmans d'Éfaté ne cherchent pas à se distinguer des autres ni-Vanuatu par des codes vestimentaires ou des signes apparents d'appartenance religieuse. Ils vivent dans un environnement social plurireligieux, où les célébrations se partagent, les gens se marient avec des conjoints d'autres confessions, certains mangent halal, d'autres élèvent des cochons, et beaucoup se rendent le soir au nakamal pour boire le kava ${ }^{19}$. La vive concurrence que se livrent des dizaines d'Églises dans ces faubourgs de Port-Vila est parfois source de divisions mais, en tant qu'associations caritatives, ces dernières contribuent également au développement et à une relative prospérité de ces communautés périurbaines dans leur ensemble.

Une vive opposition s'est manifestée entre les musulmans de Mele et ceux originaires de Tanna résidant à Port-Vila, depuis que les seconds ont revendiqué ces dernières années une place prépondérante, à la fois à Port-Vila et à l'échelle du pays. Sous la conduite de leurs deux imams, les convertis de Tanna tentent, en effet, d'imposer leur autorité sur l'ensemble de la communauté des fidèles. La plupart d'entre eux sont apparentés au groupe familial étendu du chef et patriarche Jimmy Noankan (Entretien, Iwel, 17 novembre 2017). Contrairement à leurs coreligionnaires des zones

19. La plupart des musulmans avec lesquels j'ai discuté consommaient ouvertement du kava, alors que les adventistes le font généralement en se cachant. Quelques-uns ont justifié cette liberté par le fait que la non-fermentation du kava pour sa préparation autorisait sa consommation en regard des lois de l'islam. Cheikh Mustapha me suggéra une explication alternative: «Vanuatu est tellement petit à l'échelle du monde qu'Allah avait oublié de s'y soucier de l'usage de cette plante. Non mentionné par les écritures saintes, il ne doit pas être explicitement interdit» (Entretien, 10 novembre 2017). 
périurbaines de Port-Vila, la vingtaine de fidèles originaires de Tanna rencontrés au cours de mon enquête réside généralement dans les quartiers déshérités de la ville. Souvent sans emploi, leur situation est plus précaire; les plus chanceux sont chauffeurs de bus, veilleurs de nuits ou manœuvres. Unis par des liens de parenté et d'alliance, ils bénéficient également du soutien du reste de la communauté des man Tanna de Port-Vila pour lesquels une même origine insulaire compte bien davantage que l'appartenance confessionnelle.

On peut également croiser dans les rues de Port-Vila un autre type de personnages. Il s'agit de man Tanna qui affichent ostensiblement un kufi (couvre-chef "musulman»), une barbe fournie, des cheveux coupés à ras, portent parfois une djellaba et s'apostrophent par des noms et des salutations en arabe. Pourtant, ces jeunes ne fréquentent pas la mosquée, pas plus qu'ils ne prient: "Ils se disent simplement musulmans, alors qu'ils boivent, vont au night-club, mangent du porc. Lorsqu'ils viennent à Tanna, je leur rappelle que leurs manières ne s'accordent pas avec la loi de l'islam" (Entretien avec Cheikh Mustapha, 10 novembre 2017). Tandis que la population redoute les "musulmans-terroristes", ces jeunes cherchent à en adopter le look. Autrefois, ce type de jeunes, provocateurs affichant un tempérament rebelle, était surnommé des "Rambo"; aujourd'hui on les appelle, sur le ton de la moquerie, des «Ben Laden " ${ }^{20}$. De même, quand des ni-Vanuatu musulmans affichent des signes d'identification religieuse dans les rues de Port-Vila, ils risquent de se voir qualifier d' "Arabes»"

La construction récente de stéréotypes et de préjugés sur les musulmans de Vanuatu participe d'un discours anti-islamique plus général, porté par une campagne médiatique dont nous pouvons retracer les dernières étapes de son déroulement.

\section{Le discours anti-islamique et son contexte}

Jusque dans les années 1990, à l'exception des membres des classes urbaines plus éduquées, la population de Vanuatu connaissait peu les autres religions présentes dans le monde. Néanmoins, une certaine curiosité se

20. Debra McDougall a observé les mêmes comportements chez de jeunes citadins à Honiara, la capitale des Salomon, où parmi les "styles rebelles» (tout comme à Vanuatu) figure celui du «rasta» (2009: 486).

21. La première ni-Vanuatu ayant porté le voile, en public, à Port-Vila en 2004, en témoigne: "La première fois que l'on m'a vu habillée ainsi [voilée], on m'a demandé: “D’où viens-tu?". Ceux qui m’ont posé la question étaient peut-être en colère, je ne sais pas. J'étais effrayée en revenant à Vanuatu [après un séjour prolongé en Malaisie], car ceux qui réalisaient que j'étais musulmane, ne me qualifiaient pas de "musulmane", mais d'“Arabe" " (extrait du reportage de Mark Corcoran, "Island Dress", Journeyman Pictures, 2005. En ligne: https://www.journeyman.tv/film/2611). 
manifestait couramment dans les conversations pour les magies africaines (majik blong Afrika), pour le culte rendu à un «bœuf» (wosipem buluk) par les Hindous, ou bien, au contraire, pour «l'absence de religion" prêtée aux Chinois, si ce n'est leur vénération supposée pour l'argent. En revanche, la référence à des ni-Vanuatu convertis à l'islam, les man muslam («musulmans», en bislama), est plus tardive. Communément perçu, par les non-musulmans, comme une « religion nouvelle" (wan niu relijin), l'islam est fréquemment qualifié de "dernière Église» (wan last joj) à être apparue dans le pays. À la faveur d'un niveau d'éducation plus élevé, d'échanges directs avec des convertis ou grâce à des informations diffusées par les médias, l'ignorance autrefois généralisée sur l'islam s'efface progressivement, laissant désormais la place à un foisonnement de questionnements sur la position à adopter envers sa pratique.

Cependant, nombreux furent les ni-Vanuatu à apprendre l'existence de l'islam par les attentats perpétrés aux États-Unis en septembre 2001, puis à Bali en octobre 2002. Sans ces événements et leur médiatisation, l'islam mélanésien aurait sans doute pu continuer à être perçu comme un phénomène localement marginal. C'est dans cette conjoncture sensible que les ni-Vanuatu, notamment en milieu rural, apprirent qu'un islam domestique était implanté sur leur île. La presse à Port-Vila se fit l'écho de cette découverte ${ }^{22}$, relayée par des comptes rendus dans les médias étrangers. Un article paru dans le Vanuatu Daily Post le 20 octobre $2004^{23}$ contribua à la naissance de cet intérêt soudain. Il s'agit d'une interview donnée par Mustapha Kalaos, portant sur l'histoire et la situation de l'islam dans le pays. Avec des propos rassurants, il souhaite corriger l'image négative de l'islam véhiculée par les médias. Sans préciser le nombre de convertis, il préfere insister sur des thèmes tels que la compatibilité de l'islam avec la culture locale, sa capacité à favoriser le développement et l'éducation de la jeunesse, à contribuer à l'équilibre et au bien-être des communautés par la promotion du respect et de la tolérance. Les remerciements à la fin de l'entretien donnent à penser que cette opération de communication est une réussite: "Votre article a su adopter une attitude positive pour découvrir notre belle religion et sa pratique». Cet article fut traduit et reproduit l'année suivante sur un site internet de djihadistes tchétchènes, ce qui ne fut pas sans susciter des interrogations (Flower 2017: 15).

22. Comme en règle générale, pour ce type de données, il est difficile d'évaluer précisément l'influence que les médias de Vanuatu ont pu avoir sur l'imaginaire des populations et sur leur sentiment de défiance envers l'islam. Les médias n'en nourrissent pas moins la rumeur, entendue des dizaines de fois lorsque que j'ai évoqué le sujet avec des amis peu instruits, suivant laquelle l'islam serait une "religion de terroristes».

23. "Muslims in Vanuatu», par Shirley Joy. 
Au-delà de cette anecdote, la progression de l'islam à Vanuatu devint rapidement un sujet sensible et polémique. Ben Bohane fait partie des auteurs qui se sont exprimés sur la question. Reporter photographe australien, correspondant sur place pour les médias de la région, il est membre du conseil consultatif (think tank) Pacific Institute of Public Policy, à PortVila, et a régulièrement collaboré avec le magazine Island Life. Dans deux articles à sensation publiés en 2007, Ben Bohane traite de la "question de la sécurité». L'islam y est clairement abordé comme une menace. Le risque viendrait de la contamination d'un islam pacifique, implanté à l'est de la Mélanésie, par un islam belliqueux venu d'Indonésie. Selon Bohane, les conflits entre chrétiens et musulmans ont causé la mort de plus de 10000 personnes à Ambon et dans les autres îles Moluques, à la fin des années 1990. D'après les informations qu'il a recueillies auprès de John Koknak, commandant de l'Organisation pour une Papouasie libre (Organisasi Papua Merdeka), "plus d'une douzaine de camps d'entraînement pour le jihad [seraient installés] en Papouasie occidentale, dont beaucoup proches des frontières avec la Papouasie-Nouvelle-Guinée et l'Australie». Les « $800 \mathrm{~km}$ de frontières incontrôlées de la Papouasie-Nouvelle-Guinée avec l'Indonésie [constitueraient] une porte d'entrée pour les terroristes vers le Pacifique». De nombreuses "mosquées de brousses», dans les hautes terres de PapouasieNouvelle-Guinée, pourraient servir de "têtes de pont»; "les Australiens et les Américains demeurent concentrés sur l'Iraq et l'Afghanistan, au lieu de cela, ils feraient mieux de s'occuper de leur pré carré, ici dans le Pacifique» ${ }^{24}$.

Depuis, des médias nationaux à Vanuatu reprennent régulièrement le thème d'un islam menaçant. En 2015, un article du Vanuatu Daily Post signale que le site internet de la revue Island Life Magazine a été piraté, affichant des images et slogans en faveur de l'État islamique ${ }^{25}$. Un an plus tard, Sambo, alors président du VIs, doit se justifier, dans une interview, après les accusations du Vanuatu Christian Council, selon lesquelles «l'islam entraînerait les jeunes [de Vanuatu] à devenir des terroristes ${ }^{26}$. Un autre article expose un photomontage racoleur, montrant des femmes de Tanna habillées de jupes traditionnelles en feuille, mais voilées, exécutant une danse coutumière après la prière à la mosquée ${ }^{27}$. L'année suivante, la presse rapporte les débats parlementaires au sujet d'un décret sur le contre-terrorisme et le crime transnational organisé. Le nom de Jeff Patunvanu, homme d'affaires

24. Cf. Ben Bohane, "Green Moon Rising: Islam Is Spreading In Melanesia", art. cit. (en ligne: https://web.archive.org/web/20071010082524/http://www.pacificmagazine.net/issue/2007/06/29/ green-moon-rising).

25. Cf. Dan McGarry, «Local Website Hacked», Vanuatu Daily Post, 18 novembre 2015.

26. Cf. Len Garae, "Over 1,000 Muslims», art. cit.

27. Cf. Vlad Sokhin, «Islam in Melanesia», Vanuatu Daily Post, 29 octobre 2016. 
originaire de l'île de Malekula et porte-parole du parti politique du mouvement coutumier Nagriamel, y est évoqué pour rappeler que ce dernier fut le premier insulaire du Pacifique à envoyer un message de condoléances, relayé par Al Jazeera, après l'annonce des décès de Saddam Hussein, puis de Ben Laden ${ }^{28}$. Peu de temps après, l'hebdomadaire Vanuatu Independent publie une interview de l'amiral Harry B. Harris Jr. en charge de l'Us Pacific Command, dans un article intitulé " "Wake-up Call": Us Admiral Warns of IsIs Danger in Pacific» ${ }^{29}$.

Pour se faire une idée de la méfiance de la population envers l'islam, la page de discussion Facebook la plus consultée du pays, «Yumi Toktok Stret» ("Parlons entre nous»), est un bon indicateur. Chaque évocation de cette religion y entraîne un déferlement de commentaires négatifs, méprisants voire agressifs, souvent en relation avec l'oppression des "frères mélanésiens" de la Papouasie occidentale par des "Indonésiens musulmans». Assimiler l'occupation coloniale de la Papouasie occidentale à une guerre de religion est un réflexe courant dans l'opinion publique. La plus fréquente de ces marques d'hostilité insiste sur le caractère étranger de l'islam et, par extension, de ses pratiquants. C'est sur ce point que le travail de sape contre le développement local de l'islam, qu'il émane de représentants du clergé, des réseaux sociaux ou des médias dominants, s'est montré le plus efficace pour convaincre une bonne partie de l'opinion publique. Un article du Sydney Morning Herald, intitule "Heeding the Call to Prayer in a Region that Reveres the Pig", illustre parfaitement cette rhétorique du caractère exogène de l'islam. La réponse à l'interrogation formulée par son auteur, Ben Bohane ${ }^{30}$, semble déjà contenue dans la question: «Dans quelle mesure la Pacific Way ${ }^{31}$ peut-elle se mélanger avec les enseignements d'un prophète arabe dont les visions qu'il eut dans le désert sont aujourd'hui en train de se propager dans les îles dispersées d'une mer lointaine?». L'exemple de Tanna nous donnera quelques pistes.

\section{La kastom de Tanna et le développement de l'islam}

À Port-Vila, ces dernières années, les discours visant à démontrer le caractère étranger de l'islam, son incompatibilité avec le christianisme et les valeurs de la kastom ont rencontré un certain écho parmi de larges secteurs de la population. Cette rhétorique anti-islam fut toutefois bien

28. Cf. Jane Joshua, «Patunvanu Clarifies Association With Muslims», Vanuatu Daily Post, 15 juin 2017.

29. Cf. The Vanuatu Independent, 29 juin 2017.

30. Cf. The Sidney Morning Herald, 8 septembre 2007 (en ligne: https://www.smh.com.au/world/ heeding-the-call-to-prayer-in-a-region-that-reveres-the-pig-20070908-gdr29k.html).

31. Idéologie pan-Pacifique développée dans la région à partir des années 1970. 
moins opérante à Tanna, en milieu rural, où est implantée, depuis les années 2000, la plus importante communauté musulmane de l'archipel. Une des principales raisons pour lesquelles les man Tanna se sont montrés plus ouverts à cette nouvelle religion est à chercher dans l'histoire mouvementée de la christianisation de leur île. Ils résistèrent avec détermination aux premières tentatives d'évangélisation menées à partir de 1842 par des missionnaires de la Société missionnaire de Londres (London Missionary Society). Après de premières déconvenues, leurs successeurs, les presbytériens, connurent de réels succès à partir de la fin du XIX ${ }^{e}$ siècle. La stratégie de leur congrégation, la seule alors présente sur l'île, était fondée sur une stricte intransigeance non seulement envers les croyances et pratiques païennes des habitants de Tanna, mais aussi contre presque tous les aspects de leur culture (Guiart 1956). Jusqu'à la fin des années 1930, marquées par l'arrivée dans l'île des missions adventiste et catholique, les missionnaires presbytériens voulurent encore croire en leur projet d'établir à Tanna, avec l'aide de chefs convertis, un modèle de théocratie conforme à leur dogme. Mais, plus encore que l'activité de missions concurrentes, c'est la naissance d'un nouveau mouvement religieux anticolonial, d'inspiration nativiste et millénariste, fédéré autour d'une figure surnaturelle connue sous le nom de "John Frum», qui anéantit les rêves d'hégémonie de la mission presbytérienne et bouscula les autorités administratives du Condominium (Tabani 2008).

La compétition entre missions, puis la réaction du mouvement John Frum à la tyrannie de la mission presbytérienne donnèrent à vivre à la population de Tanna une expérience religieuse singulière en matière d'innovation culturelle et d'adaptation de leurs croyances et traditions. De manière plus marquée que dans d'autres îles, l'adhésion religieuse y devint fortement conditionnée par des considérations d'alliances politiques locales. Au lendemain de la Seconde Guerre mondiale, la rébellion John Frum favorisa un éparpillement des affiliations religieuses à Tanna ${ }^{32}$. Fluctuantes et composites, elles donnèrent lieu à des assemblages toujours plus complexes avec la reprise de l'activité missionnaire étrangère dans les années 1970 et son accélération au cours des années 1990. Aux Églises déjà présentes vinrent s'ajouter des bahaïs, des apostoliques, des baptistes, des mormons, ainsi qu'une douzaine d'Églises et de communautés néo-évangéliques supplémentaires, sans compter les missionnaires évangéliques de l'Institut linguistique

32. Dans les années 1950, Jean Guiart s'employa à distinguer les «ex-chrétiens» revenus à un «strict paganisme» ou ayant adhéré aux visions «néo-païennes» du mouvement John Frum, des néo-chrétiens ou "presbytériens de principe, mais revenus aux règles de la coutume»; des "chrétiens douteux " et des groupes "coutumiers indéterminés»; des presbytériens ayant rejoint l'Église adventiste, avant de se rallier aux groupes John Frum et/ou à l'Église catholique; des païens ayant quitté le mouvement John Frum en lui reprochant l'exubérance de ses pratiques néo-traditionnelles (1956: 263 et passim). 
d'été (Summer Institute of Linguistic, SiL) engagés dans la traduction et la diffusion de la Bible. Des dissensions apparurent aussi entre des groupes traditionnalistes païens, le mouvement John Frum et de multiples courants religieux néo-païens rivaux. Compte tenu de cette fragmentation confessionnelle, la conversion d'une communauté villageoise à l'islam n'avait rien d'extraordinaire en soi. La présence de musulmans vint simplement s'ajouter à la versatilité religieuse qui règne sur l'île et aux considérations opportunistes qui motivent parfois le choix de nouvelles affiliations à des fins économiques et politiques. La multiplicité des conversions pour une même personne au cours de son existence, la fréquentation de plusieurs Églises ou de mouvements cultuels simultanément, la diversification des appartenances religieuses pour un même groupe familial afin de s'attirer les aides et la grâce de plusieurs Églises sont des choix assumés par de nombreux man Tanna. Par ailleurs, malgré les influences chrétiennes, le poids structurel de la kastom de Tanna dans les échanges cérémoniels et matrimoniaux, ainsi que la place idéologique qu'elle occupe dans l'identité insulaire demeurent socialement incontournables pour l'ensemble de la population de l'île. $20 \%$ des habitants déclarent la kastom comme seule affiliation religieuse (VNso 2009: 77), de loin le plus haut pourcentage de tout l'archipel. Le paysage religieux très diversifié de Tanna tend à y favoriser une relative tolérance.

Cette analyse sur l'islam à Tanna ne fut pas le fait de musulmans récemment convertis, mais des membres du mouvement John Frum, à un moment de son histoire où il fut traversé par un puissant renouveau millénariste. Des divisions internes avaient conduit en 2000 à une scission du mouvement en deux groupes opposés: l'un constitué autour du chef Isak Wan (héritier de la lignée historique des visionnaires John Frum), l'autre autour de Fred Nassé, un homme du commun, dont les visions eschatologiques reçurent une très large audience, et qui fut qualifié de "prophète" par ses fidèles. La rivalité entre ces deux factions prit d'abord une dimension théologique, portant sur l'interprétation à donner aux signes de la fin des temps, à l'identité christique de John Frum et à l'imminence de son second avènement. Après les attentats du 11 septembre 2001, des prises de position géo-politico-mythiques accentuèrent ces clivages. Les interventions de l'armée américaine en Afghanistan, puis en Irak, amenèrent Nassé et ses partisans à vouloir remettre en cause les supposées relations privilégiées avec les États-Unis, dues au fait que l'armée américaine aurait protégé la kastom pendant son occupation de l'archipel au cours de la Seconde Guerre mondiale (Tabani 2008). Nassé se prononça donc pour le rejet des pratiques rituelles philo-américaines du mouvement, que perpétuaient son adversaire Isak Wan et ses partisans. 
Celui-ci ne fut pas le dernier à commenter ces sujets d'actualité pour maintenir son influence, alors que des slogans pro-Ben Laden firent leur apparition sur les murs en tôle de quelques maisons et que des chants John Frum furent composés en son honneur. Devant l'affirmation d'un sentiment anti-américain à Tanna, Isak Wan apporta une réponse de théologien. Au cours de plusieurs entretiens en 2002 et en 2004, il m'exposa son interprétation des entreprises bellicistes de l'Amérique et de leurs implications symboliques pour son mouvement. Son exégèse des événements du monde, plus ésotérique encore que celle de son rival, contribua à faire connaître, à Tanna, l'existence de l'islam en tant que religion coutumière des man muslam. Le soupçon d'un lien entre John Frum et Ben Laden se répandit de Tanna jusqu'à Port-Vila, au sein de la population locale et parmi le petit monde des résidents étrangers, des expatriés en particulier. Selon Isak Wan, l'Amérique ne saurait être mauvaise, en vertu du lien entre Tanna et les États-Unis. La raison pour laquelle cette puissance s'en prenait à des man muslam qui, tout comme les man Tanna, ne feraient que protéger leur kastom, serait à chercher ailleurs. Le président américain aurait été possédé par Nakua (Satan), éternel ennemi de John Frum. Pour libérer George W. Bush de cette emprise, John Frum aurait investi un saint homme, Ben Laden, un vaillant défenseur de la coutume auquel il aurait transmis des pouvoirs. Sans l'aide des pierres magiques de Tanna qui lui furent remises, cet homme n'aurait pas été en mesure à lui seul de frapper l'Amérique, ni de disparaittre impunément sans être retrouvé (Entretien avec Isak Wan, Lamakara, février 2002 et 2004).

Quoi qu'il en soit, cette agitation au sein du mouvement John Frum devait avoir pour conséquence de gêner pour un temps le prosélytisme plus orthodoxe de Jimmy Noankan.

\section{Jimmy Noankan, patriarche de la communauté musulmane de Tanna}

Son cheminement personnel pour devenir le patriarche de la communauté islamique de Tanna a été facilité, en partie, par son statut de chef dans la "coutume" (iani niko) et, par là même, par l'histoire de son groupe territorial (niko, parfois imparfaitement traduit par «tribu») des Nalhyaone. L'histoire coloniale des familles de son niko, l'un parmi la centaine que compte la société de Tanna, fut marquée par leur abandon précoce de la mission presbytérienne et leur retour à la coutume. Ils s'engagèrent ensuite au sein du mouvement John Frum, avant de préférer s'allier à des groupes plus stricts dans leur conception de la coutume, connus sous le nom de mouvement kapiel (Bonnemaison 1996), en référence aux pierres de magie au fondement de la cosmologie de Tanna. 
Jimmy Noankan est un héritier des Nalhyaone et de leurs stratégies complexes, faites de revirements politiques et religieux, pour maintenir leur influence. Encore trentenaire, il abandonna les croyances suivies par son père pour rejoindre, avec des membres de son niko, l'Église adventiste. Parallèlement, il s'engagea dans la lutte pour l'indépendance aux côtés du Vanuaaku Pati (VP), parti nationaliste, dont il devint l'un des cadres à l'échelle locale. À la fin des années 1970, Tanna, comme le reste du pays, connut une montée des tensions qui divisa la population entre une partie alignée sur les positions de la France, qui souhaitait différer leur pleine souveraineté qu'elle jugeait précipitée, et une autre, soutenant les préférences de la Grande-Bretagne pour une transition rapide vers l'indépendance. Noankan quitta alors le Vp pour rejoindre le camp politique des groupes et partis pro-français (composé localement de groupes John Frum, catholiques et coutumiers). Au cours des années 1990, il prit ses distances avec les adventistes et rejoignit une confédération de traditionalistes dite des «Douze nakamal» 33 (Tabani 2019). L'engagement de Noankan pour un retour vers une coutume intégrale ne s'accordait ni avec les prophéties des pentecôtistes d'une apocalypse imminente ni avec l'attente salvatrice d'un second avènement de John Frum. L'islam lui parut alors plus adapté aux exigences de la coutume et à des représentations sotériologiques plus conventionnelles. Toutefois, il ne pouvait pas s'attendre à ce qu'un événement mondial de l'ampleur du 11 septembre 2001 braque les projecteurs médiatiques sur sa nouvelle religion.

Une fois découvert, l'islam devint à Tanna l'objet de conflits d'interprétations et d'enjeux théologiques et idéologiques. Noankan se devait d'affirmer son point de vue et sa légitimité. En tant que patriarche de sa communauté, il réussit à s'imposer comme expert exclusif pour toutes les questions concernant l'islam. Il dénonça les spéculations des groupes John Frum comme étant fantaisistes. Mais il dut en même temps les ménager, du fait de leur alliance pour la défense de la kastom, quel qu'en soit leur degré d'hétérodoxie. Sans compter que l'islam ne cessait d'attirer d'anciens membres du mouvement John Frum (qui ont également fourni, dans les années 1980, une bonne partie des membres de la communauté bahaïe de l'île) ${ }^{34}$. Il n'hésita pas, d'ailleurs, à accoler à son nom un grade symbolique, à la manière des leaders John Frum: «Kapten» Naïm Noankan.

33. Nakamal, en bislama, désigne, à Tanna, la place cérémonielle où s'expriment publiquement les autorités coutumières.

34. L'implantation des bahaïs à Tanna (deux à trois fois plus nombreux que les musulmans) a suivi un cheminement comparable à celui de l'islam. D'après Graham Hassall: «Les croyances bahaïes ne sont pas nécessairement opposées à celles de la coutume, et certaines grandes concentrations de bahaïs se sont formées dans des îles comme celle de Tanna à Vanuatu et Malaita aux îles Salomon, où la coutume est demeurée particulièrement forte. Ce qui ne veut pas dire, d'un autre point de la vue, 
Sa persévérance lui permit de faire reconnaître qu’à Tanna, lui seul et son groupe disposaient d'un monopole sur les alliances avec les pays musulmans.

Sa stratégie s'avéra payante puisque depuis vingt ans, la communauté musulmane de Tanna ne cesse de croître. Les musulmans de Tanna tirent de nombreux avantages matériels de leur nouvelle religion. Ainsi, par exemple, ils bénéficient régulièrement de voyages et de bourses d'études, de financements pour des projets de développement (une école est en construction, à Iwel, et une autre en projet, à Lamnatu), et de la présence de bénévoles étrangers pour en assurer la gestion. L'aide humanitaire qui a suivi les destructions du cyclone Pam en 2015, abondamment médiatisée par les ONG islamiques de la région (Muslim Aid Australia, Fijian Muslim League, Rasheed Memorial Dawah Trust [Nz]), ainsi que par la Turquie, fut tellement conséquente, qu'elle amena momentanément à multiplier par trois le nombre de convertis à Tanna. Cette réalité n’a pas échappé au missionnaire Tom Richards, comme en témoigne cet extrait de son article publié sur son blog 35 , en juillet 2015 :

«À en croire les gens, [l'aide apportée par des ONG islamiques] supposait [...] de remplir un formulaire stipulant une adhésion à l'islam. Ce formulaire ambigu n'indiquait pas clairement qu'il fallait le signer pour obtenir du riz. Toutefois, pour la mentalité de Tanna qui tend à voir la religion comme une route pour obtenir des biens matériels et établir des relations à cette fin, cette signature impliquait une promesse. Le pasteur presbytérien Dino au Centre-Brousse indiqua que le nombre de gens assistant à la prière musulmane, d'anciens adventistes pour la plupart, avait diminué avec la fin de l'aide. Mais certains sont restés musulmans et les autres ne retourneront jamais à leur ancienne Église».

Les musulmans (à l'instar des mormons) peuvent ainsi être parfois accusés d'une sorte d' "opportunisme» religieux, leur réussite matérielle en apportant la preuve. Le père Nathaniel, ancien prêtre de la paroisse catholique de Loanatom, se souvient avoir été souvent surpris lorsqu'il venait réceptionner sur le quai des marchandises livrées par les cargos. Le petit groupe de musulmans recevait des livraisons plus importantes que celles destinées à l'ensemble des nombreux catholiques de la mission (Entretien, Port-Vila, 16 octobre 2017). Un élu de Tanna, administrativement en charge des affaires religieuses locales, me décrivit, sur un ton désabusé, les motivations des adeptes de nouvelles religions en ces termes:

[Suite de la note 34] que les lois coutumières seraient en parfait accord avec les lois bahaïes, puisque les premières ont été adaptées pour une part, dans le contexte du Pacifique, aux secondes» (2005: 282). 35. Cf. Tom Richards, "Faith That's Clothes Deep», Talking About Tanna (en ligne: https:// talkingabouttanna.com/2015/07/20/faith-thats-clothes-deep/\#more-623). 
«Le christianisme s'est imposé depuis longtemps, partout à Vanuatu. L'Église l'a enseigné à tout le monde. Aujourd'hui, à Tanna, les gens se tournent vers de nouvelles Églises uniquement par intérêt matériel. Une Église qui a de l'argent, ils vont la suivre. Pour obtenir des adhésions, elles doivent dorénavant construire des bâtiments, acheter une voiture, rendre des services, et les Blancs qui les dirigent le savent bien. Le problème est plus exacerbé à Tanna, car ses habitants ont toujours été matérialistes et individualistes, avant même la venue des Blancs» (Anonyme, Lenakel, 5 décembre 2017) ${ }^{36}$.

Les musulmans de Tanna expliquent leur prospérité économique par les spécificités de leur religion. Son originalité reposerait sur l'égalité entre tous ses fidèles. À l'inverse des divisions et des rivalités qui agitent les congrégations chrétiennes, l'islam impliquerait, selon Jimmy Noankan, une fraternité généreuse, dans laquelle n'interviendrait aucune considération hiérarchique ni volonté de domination:

"Quand nos frères musulmans de l'étranger viennent nous rendre visite, ils partagent nos repas en mangeant avec la main; ils nous montrent ainsi que nous sommes égaux, aucun ne prétend être plus important, ne se revendique ministre ou pasteur » (Entretien, 18 février 2008).

Ces considérations ne sont pas dépourvues de certaines implications politiques et idéologiques. Pour Noankan, la morale universelle que promeut cette religion la distingue clairement de l'ambition affichée par l'Amérique et ses soutiens dans le monde anglo-saxon, qui cherchent, quant à eux, à «s'emparer des ressources des peuples noirs» (Id.).

S’imposant comme le chantre d'une négritude mélanésienne, le patriarche Jimmy Noankan tient à souligner les affinités entre l'islam et les valeurs françaises. Parce qu'elle aurait protégé, par le passé, la coutume de Tanna, la France est réputée être plus apte à fournir une aide au développement respectueuse des traditions locales. Ses citoyens noirs et musulmans, en outre, disposeraient des mêmes droits et bénéficieraient des mêmes avantages que les autres Français, tandis que l'Amérique aurait pour habitude de discriminer ces minorités. Le lien entre la France et Tanna serait antérieur à celui établi autrefois avec l'Amérique et John Frum, à une époque où ce puissant pays protégeait la liberté des peuples à disposer de leurs coutumes. L'affinité avec la France dépasserait le cadre d'une alliance opportuniste;

36. Comme l'explique Tom Richards : "Offrir des biens matériels ne constitue pas une nouvelle stratégie missionnaire à Tanna. Les Mormons ont pour habitude de payer les frais de scolarité pour les enfants dont les parents acceptent de rejoinder leur Église. Une offre qui n'est pas réservée aux groupes non chrétiens. Il n'est pas inhabituel pour les membres de notre mission de rejoindre d'autres obédiences, car ils y trouvent un avantage. Ainsi, notre ancien champ missionnaire [à Tanna] est désormais accaparé par les mormons qui paient les frais de scolarité, les apostoliques qui proposent des adductions d'eau, par [l'Église] Upper-room qui offre des citernes, et désormais également par l'islam. Cela multiplie les parts pour un petit gateau communautaire», cf. note 35, art. cit. 
il serait consubstantiel, familial. D'après Noankan, la France et Tanna partagent des ancêtres en commun: Tanna est l'île d'origine des Français qui la quittèrent dans des temps anciens:

«La France est à la tête de la route d'alliances avec l'Amérique. Mes ancêtres qualifiaient notre lien avec l'Amérique de nuk nanimien, en référence à un arbre qui glisse comme du savon. S'il n'y a plus d'autres routes praticables pour l'Amérique, cette dernière doit s'en remettre à la France pour la ramener sur la bonne voie. La France est la vraie porte. Nous savons par ailleurs qu'il y aura prochainement un président noir au États-Unis, et nos chefs voient cela comme le signe que les Noirs doivent se saisir du pouvoir. Quand c'est le Blanc qui dirige, il abîme tout, cause de nombreux problèmes dans le monde. Sa loi n'est pas en harmonie avec la kastom. Il décide qu'il faut voter pour que notre kastom puisse revenir mais, en réalité, la coutume a perdu ses pouvoirs. Elle reste présente dans nos cœurs, alors qu'elle a disparu dans la pratique. Dans le monde, tous les musulmans, tous les Noirs veulent maintenir la coutume, mais l'Amérique que l'on appelle aussi Us envoie ses avions vers l'est, vers l'autre bout du monde pour combattre, pour s'emparer des ressources des Noirs, des ressources que Dieu a données aux Noirs pour qu'ils puissent vivre. Seul le Blanc revendique à chaque fois qu'il est le roi, qu'il va être le maître, et menace de nous rabaisser. L'histoire des musulmans signifie qu'il va y avoir un changement. Si tu vas quelque part et que tu frappes quelqu'un jusqu'au sang, tu ne peux plus retourner chez lui» (Id.).

L'Amérique causerait aujourd'hui les mêmes torts aux musulmans que ceux infligés autrefois par les presbytériens aux coutumiers, ce qui incite Noankan à exprimer ouvertement des propos anti-presbytériens, afin de montrer qu'il ne les craint nullement:

«Au temps de la Tanna Law [Loi de Tanna], les presbytériens avaient déjà tenté de tuer la coutume [...] ils contaminaient avec de l'acide les sources d'eau des hommes des groupes coutumiers qui, après avoir bu de cette eau, avaient du sang dans leurs déjections et perdaient leurs dents. Heureusement, la France est venue nous sauver et désormais nous sommes fidèles à la France et non pas à l'Australie qui soutient les presbytériens, notamment le pasteur Nafuki, président du Vanuatu Christian Council, qui est le pire d'entre eux. Il a reçu de l'argent et a été missionné par le gouvernement pour défaire la coutume ou les croyances en John Frum. À notre égard, son refrain favori est de nous accuser d'être des terroristes. Le vrai problème des presbytériens est que leur Église est en train de disparaitre, tout le monde la quitte» (Id. $)^{37}$.

37. Cette affirmation de Jimmy Noankan traduit l'opposition courante à Tanna entre la kastom, source d'unité, et l'État, facteur de division, auquel l'Église presbytérienne est historiquement associée. L'islam permet de renforcer la capacité de la kastom à contrarier l'hégémonie d'un gouvernement central. Cette situation est fort différente de celle des musulmans en Australie où, à l'inverse, dans un contexte "post-sécularisé», l'intégration des lois islamiques (shari’a) doit être en conformité avec les lois australiennes, traduisant ainsi l'affaiblissement du caractère réfractaire des religions populaires envers l'État, tel qu'il fut envisagé par Gramsci (Possamai 2016). 


\section{L'islam comme retour vers la kastom}

Alors que la prospérité économique et l'influence politique des musulmans sont régulièrement soulignées, les raisons strictement doctrinales ou théologiques pour expliquer leur adhésion à l'islam sont peu mises en avant. Or, pour les musulmans de Tanna, l'islam est vérité, puisque le Coran a retranscrit littéralement la parole divine, tandis que les Églises chrétiennes réécrivent constamment la Bible, la corrigent dans un sens qui leur convient:

"J'ai choisi l'islam car il m'offre une bonne relation avec Dieu et respecte la coutume. Je pense, que seuls les musulmans peuvent espérer rejoindre le paradis, car ils ne brisent aucune loi, ils suivent toutes celles qui sont mentionnées par le Coran et par la Bible. Autrefois j'étais Sda, une religion qui suit un peu la loi. Mais aujourd'hui, elle veut changer la loi. J'ai pu voir dans la version King James de la Bible, qu'elle contient beaucoup d'erreurs. Parce qu'en fait, chaque année ils réécrivent la Bible, alors que le Coran ne change pas. Dieu l'a dit, "mes mots ne doivent pas changer". C'est pour cela que j'ai quitté les Sda» (Entretien avec Jimmy Noankan, 17 novembre 2017).

Les musulmans de Tanna justifient le choix de leur conversion pour des raisons d'ordre moral, rituel, social ou idéologique, en dépit des obstacles culturels qui peuvent se présenter. Tous ceux avec lesquels j'ai pu discuter reconnaissent, à un degré ou à un autre, qu'il est parfois difficile de suivre strictement les prescriptions islamiques de nature alimentaire (consommation du cochon et du kava), vestimentaire (en référence, par exemple, au port du voile intégral qu'ils ont pu observer chez des hôtes saoudiennes) et contre les croyances traditionnelles (usage des magies agraires, survivances du culte des ancêtres). Le manque de formation et l'incapacité à lire le Coran sont également présentés comme des freins à une pratique plus conforme. Toutefois, les traditions héritées ne sont pas une gêne à l'accomplissement vertueux de la foi musulmane, comparées aux pratiques immorales importées de l'étranger: l'alcool, les drogues, la criminalité, la prostitution.

D'après Mustapha, un proche de Noankan: «On trouve aujourd'hui à Tanna beaucoup de marijuana, il existe de nombreux criminels, des jeunes veulent reproduire ce qu'ils peuvent voir dans des films ou sur Internet. L'islam, grâce aux nombreux projets communautaires qu'il permet de développer, est un moyen efficace pour contrer ces mauvaises influences extérieures" (Entretien, 10 novembre 2017). Et pour l'imam Abdul Karim: «De nombreux ni-Vanuatu ont adopté des façons étrangères, ils ont absorbé beaucoup de choses provenant de cultures différentes, de sorte qu'il est aujourd'hui bien difficile de maintenir ce qui existait auparavant. La priorité est de protéger la kastom» (Entretien, $1^{\text {er }}$ décembre 2017). Lislam et la kastom auraient pour objectif commun de rejeter certaines innovations nuisibles de la modernité, tâche dans laquelle les Églises chrétiennes auraient échoué. 
Cette compatibilité de l'islam avec les traditions de Tanna découlerait de leur qualité de religions coutumières (kastom relijin). Mais, plus encore, leurs pratiques se ressembleraient étonnamment: comme "tous les musulmans", les man Tanna pratiquent des sacrifices d'animaux, la circoncision, la polygamie, la séparation des sexes dans divers contextes, notamment rituels, prônent la décence et la pudeur dans les règles de conduite, en particulier pour les femmes, le port de la barbe pour les hommes. Le trait peut sembler forcé, néanmoins, avec l'affirmation des similitudes et l'occultation des innombrables différences, l'analogie qui est faite nourrit l'idée d'identité 38 . Les activités liées à la coutume seraient en tous points semblables à celles de l'islam. Ce ne fut d'ailleurs pas avant de l'avoir vérifié, et indépendamment d'autres considérations, que Noankan décida de se convertir:

«Mohamed [Ahmadu] parla de l'islam à Tom, l'un de mes fils. Il lui expliqua comment prier, comment sonner la cloche - ou faire l'adhan [l'appel à la prière] - pour que tout le monde puisse entendre l'appel à la prière, comment s'habiller, manger et se comporter avec les autres. Quand Tom est venu ici, il m’a demandé ce que je pensais de cette religion. J'ai alors convoqué deux de mes pères, pour que nous décidions ensemble quoi lui répondre. L'un des deux, qui était un chef, a conclu que cette religion était identique à notre propre kastom. Je suis donc parti pour Port-Vila, trouver Mohamed [...]. Lorsque, ensuite, il est venu nous rendre visite ici, j'ai bien fait attention à la manière dont il priait, d'une manière tranquille, sans boire de vin et sans utiliser des cassettes audio, et j'ai pu ainsi vérifier que ses façons étaient parfaitement en accord avec la kastom de Tanna. J'ai alors accepté de devenir musulman» (Entretien, 17 novembre 2017).

\section{L’islam dans la cosmogenèse des musulmans de Tanna}

La rencontre de Noankan avec l'islam est une forme d'adhésion à une religion proche de la coutume. Dès lors, la pratique de l'islam se trouve facilitée par une application intégrale de la kastom et inversement. Sur le constat d'une remarquable convergence entre coutume et religion, d'un esprit commun, l'idée s'est forgée, chez des musulmans de Tanna, que l'islam trouvait sa source en Mélanésie et, plus spécifiquement encore, dans leur propre île. L'islam ferait partie de la coutume, de toute éternité, selon l'imam Abdul Karim:

«Les manières des gens de Tanna, leurs façons de se comporter, d'agir, sont totalement en phase avec la voie de l'islam. Ce qui signifie que l'islam n'est pas une nouvelle religion,

38. Cette stricte identification entre religion (importée dans le cas de l'islam) et traditions culturelles (ancestrales), revendiquée par la communauté musulmane de Tanna, nous permet de mesurer combien ces conceptions collectives relatives à l'idée de conversion diffèrent du cheminement individuel de sujets croyants dans des pays dits «occidentaux", où prime souvent le besoin de distinguer « religion " et "culture" (cf., à ce sujet, la présentation du numéro spécial des Archives de sciences sociales des religions, "Conversions à l'islam, culture et religion : tensions et articulations" (Galonnier et al. 2019). 
mais au contraire la première dans l'histoire de l'humanité. En examinant correctement les choses, on peut s'apercevoir que nous autres, à Tanna, à Vanuatu, aux îles Salomon ou en Papouasie Nouvelle-Guinée, nous étions déjà des musulmans, et ce n'est que plus tard que les Arabes ont été influencés par notre kastom» (Entretien, $1^{\text {er }}$ décembre 2017).

D'après Scott Flower, cette assimilation de la kastom avec l'islam découlerait du concept de «réversion", présent dans la théologie islamique et employé avec un certain zèle:

"L'utilisation du concept de réversion, parmi les convertis en Papouasie-Nouvelle-Guinée, pourrait être prévalant car, en amont ou à la suite de la conversion, des missionnaires islamiques leur ont parlé du terme fitra ("réversion/réverti") et des similarités entre les coutumes» $(2017: 50)$.

Personnellement, il me semble que le type d'interprétation, qui place Tanna au commencement du monde et assigne une antériorité à la coutume sur toute autre forme de révélation spirituelle, est caractéristique des représentations des man Tanna concernant l'histoire en tant que changement culturel. Comme ailleurs en Mélanésie (Timmer 2015) ou à Vanuatu, certains habitants de Tanna affirment être des Hébreux, des descendants de la tribu perdue d'Israël, une origine sur laquelle avaient déjà spéculé des missionnaires au XIX $x^{e}$ siècle (Newland \& Brown 2015: 251). Mais, dans le cas présent, ce sont les Hébreux qui sont vus comme des descendants des Man Tanna, et non l'inverse. Les histoires bibliques et leur temporalité ont été transposées par la mythologie John Frum à l'environnement local. À en croire un informateur de Debra McDougall, les musulmans de Malaita, une des îles Salomon, vont encore plus loin dans cette assimilation entre islam et kastom, en identifiant leurs propres ancêtres à des "Arabes»:

«Après avoir migré vers les îles, ces ancêtres [des gens de Malaita] se retrouvèrent dans une confusion, au lieu de célébrer Dieu, ils vouèrent un culte aux morts. L'évangélisation chrétienne [...] était une bonne chose, en ce qu'elle leur a permis de revenir au monothéisme et à l'adoration du vrai Dieu. Au cours de ce processus, furent toutefois détruits les anciens tabous qui subsistaient de la loi islamique [primordiale]» (2009: 487).

Adam et Ève, selon une exégèse syncrétique connue de tous les man Tanna, sont originaires de leur île et vivaient sur l'actuelle plaine de cendres, au pied du volcan Yasur, assimilé au jardin d'Eden. Selon les croyances locales, le mont Tukosmera, point culminant de l'île, est également le lieu de départ de l'arche de Noé, un man Tanna. Cet ancêtre a emmené avec lui, puis dispersé sur d'autres continents, tous les grands animaux qui peuplaient autrefois l'île. Les outils qui servirent à construire l'arche furent abandonnés dans les collines derrière le volcan, près de la place de danse d'Embuitoka, où l'on peut contempler aujourd'hui encore leurs reliques pétrifiées. Pour 
avoir désobéi à Moïse, les gens de Tanna ont été dépossédés des pouvoirs dont disposent aujourd'hui les Blancs. C'est pour les consoler que Jésus leur a donné le kava.

En admettant que le prophète John Frum rappelle le personnage de Jean le Baptiste, il s'apparenterait aussi à Abraham, si l'on s'en tient aux prénoms de ses enfants, Isaac, Jacob (Guiart 1956: 159); Lastwan, le plus local d'entre eux, a inspiré le nom du chef Isak Wan. D'autres communautés religieuses prétendent pareillement avoir des liens directs avec l'univers biblique. Tel est le cas des bahaïs qui, comme d'autres Tannais, sont considérés comme des Hébreux, avec cependant la particularité de s'en tenir uniquement aux prescriptions de l'Ancien Testament. Ils sont, de ce fait, qualifiés de man jiu (juifs), des «enfants d'Abraham». Plus récemment, en l'an 2000, les partisans d'un autre prophète, Fred Nassé, ont entraîné près du quart de la population sur les hauteurs de la montagne Yenkahi, derrière le volcan, pour reconstruire l'arche de Noé, rebaptisée «Pirogue de l'an 2000 » en prévision d'un nouveau déluge. Ils ont nommé leur quartier général «La Nouvelle Jérusalem», et "Jourdain» la rivière au pied du volcan.

Ces représentations, qui situent la coutume de Tanna dans une antériorité mythologique plus ancienne que toute autre tradition culturelle et religieuse, pourraient être qualifiées de "cosmocentriques». L'adoption d'influences extérieures, sous la forme d'une appropriation ancestrale, préexistait sans doute à l'arrivée des missionnaires. Mais avec le christianisme, cette tendance s'est vue complétée, par un ethnocentrisme millénariste ou eschatologique. Le retour de John Frum à la fin des temps s'effectuera dans la même île que celle que Noé a quittée lors du déluge. Les musulmans de Tanna espèrent, à leur manière, que l'islam les ramènera, à l'avenir, vers l'état spirituel et la condition sociale qui caractérisaient, au commencement des temps, le "paradis de la coutume». Cette restauration des règles ancestrales rendra aux descendants mélanésiens les pouvoirs dont ils sont les détenteurs légitimes. L'islam à Tanna ne rejette ainsi aucun des héritages culturels ni aucune des croyances religieuses qui l'ont précédé. La coutume, le christianisme ou le culte John Frum sont autant de sources admises d'un savoir ultime vers lequel l'islam, à son tour, doit permettre de converger, en dépassant leurs oppositions et contradictions.

Revenons sur les appropriations politiques des phénomènes religieux qui ont marqué l'histoire culturelle de ce pays. J'ai évoqué plus haut comment des principes religieux et des éléments culturels initialement importés, ou, pour le moins, fortement conditionnés par des influences extérieures, ont été systématiquement opposés les uns aux autres pour justifier ou 
contester leur légitimité respective. Or, sur ce point, la propagation des mouvements indigénistes ou "cultes du cargo", l'instrumentalisation idéologique de la kastom et l'adhésion à l'islam ont fait l'objet, avant ou après l'indépendance, d'un même degré d'hostilité de la part des autorités du pays. Ce rejet fut systématiquement motivé par l'origine étrangère des influences religieuses contestées.

Ainsi, pour les autorités administratives coloniales comme pour les missionnaires, les "cultes du cargo" étaient antithétiques à l'entreprise d'évangélisation. Le mouvement John Frum, entre autres, s'apparentait à leurs yeux à une tentative de réinstauration du paganisme. Malgré ses nombreuses références bibliques, ses membres prônaient un retour vers la kastom en termes de «retour vers les ténèbres». Dans ces circonstances, aucune compatibilité entre ce mouvement et les principes chrétiens ne semblait envisageable. Quelques décennies plus tard, le pasteur et Premier ministre Walter Lini précisa la position officielle du premier gouvernement à l'égard de mouvements politico-religieux dissidents. John Frum, «véritable culte du cargo " (1980: 48), traduirait une errance spirituelle propre à des groupes présentant "des troubles dans leur adaptation aux influences occidentales" (Ibid.: 46). La continuité avec la kastom dont ils se réclament n'offre aucune ouverture vers un système démocratique moderne. Le myth-dream forgé par ces activistes de la coutume ne serait en rien chrétien (Ibid.: 48). L'usage politique qu'ils font de la kastom ne serait pas non plus mélanésien du point de vue culturel; sous couvert de références à la tradition, ils se livreraient à des «activités terroristes» (Ibid.: 53).

Quant à l'islam, il cumule aujourd'hui les deux principaux griefs imputés, par le passé, aux "cultes du cargo", en tant que responsables présumés de la politisation de la kastom qui l'aurait pervertie. Pour une partie des représentants de l'État et des Églises activement engagées dans une campagne publique contre la diffusion de l'islam dans l'archipel, cette religion ne présenterait aucune affinité avec la kastom, du fait de sa profonde altérité culturelle. Aucune proximité ne semblerait non plus être admise avec le christianisme, dès lors que l'islam est perçu comme un mouvement politique qui se draperait des atours d'une religion et ne se priverait pas d'user de violence pour nourrir sa volonté de puissance. Son extranéité lui interdirait toute capacité d'adaptation; sa diffusion ne pourrait mener qu'à une rupture irrémédiable avec une identité nationale reposant sur des héritages culturels officiellement reconnus.

Lors de l'apparition des premiers mouvements religieux de contestation en Mélanésie pendant la période coloniale, la répression fut parfois évitée grâce à l'intervention des anthropologues. Ce fut le cas à propos de Vailala Madness, un mouvement millénariste apparu dans le golfe de Papouasie, 
à la fin des années 1910. Tandis qu'il apparaissait aux yeux des autorités coloniales comme une religion déviante, voire une pathologie collective, pour l'anthropologue et spécialiste en sciences des religions, Robert Marett, il s'agissait bien d'un «mouvement religieux" à part entière. Ce dernier réussit à convaincre les autorités coloniales que, compte tenu du "précédent malheureux de Ponce Pilate", les répercussions d'une répression pouvaient avoir comme conséquence de lui donner une dimension politique (1928: $\mathrm{XI})$. Walter Strong suggéra à son tour une comparaison avec les "grandes révélations théologiques" du passé (1923: Ix). Vailala Madness prenait l'apparence des "débuts d'un credo et d'un rituel», similaire au "mahométisme qui, dans ses premiers jours, débuta dans ce type d'atmosphère psychologique» (Ibid.).

Ces «mouvements religieux de liberté et de salut des peuples opprimés» (Lanternari 1962) partageaient nombre de caractéristiques avec les religions abrahamiques. Leurs orientations millénaristes traduisaient une volonté de rompre avec le passé en vue de refonder la société. Si les mouvements dits «cultes du cargo" ont été associés aux manifestations religieuses des débuts du christianisme et de l'islam, ils auraient également inspiré des phénomènes religieux plus récents. Selon Annelin Eriksen, les Églises pentecôtistes emprunteraient beaucoup de traits autrefois attribués aux "cultes du cargo». En tant que "mouvements sociaux qui critiquent le présent», elles étaient mues par une volonté de "créer de nouvelles unités ici et maintenant»: "rompre avec le passé est leur mantra» (2009: 68). Tandis que les Églises pentecôtistes reprendraient à leur compte des objectifs antérieurement affichés par les "cultes du cargo", Debra McDougall (2009), puis Scott Flower (2017) ont suggéré, pour leur part, que la diffusion de l'islam en Mélanésie poursuit une stratégie semblable à celle, précédemment déployée par les Églises pentecôtistes. En quête de rupture avec ces dernières, les musulmans insistent sur la proximité entre l'islam et les traditions mélanésiennes, ainsi que sur le partage d'ambitions spirituelles, par opposition aux «mentalités cargoïstes» enfermées dans des convoitises purement matérielles (Flower 2015).

S'appuyant sur les points communs, dans les modes d'action, entre doctrines concurrentes soulignées par les anthropologues cités ci-dessus, Ben Bohane entrevoit, quant à lui, avec moins de nuances que ces derniers, un lien direct entre les succès rencontrés par l'islam en Mélanésie et ceux remportés autrefois par les "cultes du cargo ». Les deux phénomènes partageraient des sensibilités et des enjeux similaires:

«L'islam pourrait bien devenir le prochain "ingrédient" déterminant dans le potpourri de la croyance religieuse en Mélanésie et influencer l'évolution des kastom et de 
divers mouvements cultuels, surtout si ces derniers se voient soutenus dans la capacité "d'apporter le cargo" [i.e. les biens que les Blancs détiendraient indûment] au sens à

Les craintes soulevées par une croissance de l'islam à Vanuatu pourraient, dans ces conditions, être justifiées. Car, à la différence des attentes illusoires des «cultes du cargo" d'autrefois, les pays et organisations qui soutiennent la diffusion de l'islam disposeraient des capacités matérielles pour concrétiser les rêves d'abondance des nouveaux convertis, et seraient par là même susceptibles de générer un prosélytisme de forte ampleur.

Les développements de l'islam à Tanna nous présentent toutefois un tableau bien différent, d'où son intérêt comparatif. Dans cette île souvent décrite comme une Mecque des "cultes du cargo", l'usage des notions de discontinuité et de rupture, telles qu'elles ont été employées par exemple par Joel Robbins (2007) pour analyser les changements religieux radicaux, paraissent trop larges pour s'appliquer aux enjeux d'une profonde mélanésianisation d'influences religieuses extérieures (Barker 2007: 18). Ladhésion à l'islam des man Tanna ne peut se réduire aux seules convoitises matérielles que laisse suggérer une analogie avec les "cultes du cargo". Leur indéfectible volonté de renouer avec le fil de la tradition les a rendus maitres dans l'art de rajeunir leurs mythes et de rafraîchir leurs pratiques ancestrales. Donner au changement une apparence de permanence et jouer sur l'illusion d'une continuité ont été les moyens privilégiés pour intégrer des traditions religieuses étrangères et inédites à leur passé.

Centre national de la recherche scientifique, Centre de recherche et de documentation sur l'Océanie, Marseille marc.tabani@pacific-credo.fr

MOTS CLÉS/KEYWORDS: islam - christianisme/Christianity - Vanuatu - île de Tanna/Tanna Island - culte du cargo/Cargo cult- John Frum - kastom - changement culturel/cultural change. 


\section{RÉFÉRENCES CITÉES}

Ahmadu, Mohammed Lawal

\& Zainab M. Shuaibu

2004 A Short Introduction to Islam and Muslims in Vanuatu. Kuala Lumpur, Regional Islamic Dawah Council of South East Asia and the Pacific (RIseap).

Ali, Jan

2004 "Islam and Muslims in Fiji", Journal of Muslim Minority Affairs 24 (1): 141-154.

Barker, John

2007 "Comments to "Continuity Thinking and the Problem of Christian Culture:

Belief, Time and Anthropology of Christianity" ", Current Anthropology 48 (1) : 18 .

\section{Bohane, Ben}

2007 Blackfella Armies. Kastom and Conflict in Contemporary Melanesia 1994-2007.

Wollongong, University of Wollongong, MA Thesis.

Bonnemaison, Joël

1996 Les Fondements d'une identité. Territoire, histoire et société dans l'Archipel de Vanuatu (Mélanésie). Paris, Orstom ("Travaux et documents» 201).

\section{Crowley, Terry}

1995 A New Bislama Dictionary.

Suva, Fiji, Institute of Pacific Studies/

Vila, Vanuatu, Pacific Languages Unit.

\section{Eriksen, Annelin}

2009 «Healing the Nation:

In Search of Unity through the Holy Spirit in Vanuatu", Social Analysis 53 (1): 67-81.

\section{Eriksen, Annelin \& Rose Andrew}

2010 Churches in Port Vila.

En ligne: https://genpent.w.uib.no/

files/2012/11/Churches-in-Port-Vila-ferdig.pdf

\section{Flower, Scott}

2015 "Conversion to Islam in Papua New Guinea: Preserving Traditional Culture Against Modernity's Cargo-Cult Mentality", Nova Religio 18 (4) : 55-82.

2017 Islam and Cultural Change in Papua New Guinea.

London-New York, Routledge.

Galonnier, Juliette et al.

2019 "Conversion à l'islam: introduction", Archives de sciences sociales des religions 186 :

11-31.

Graille, Caroline

2015 Des militants aux professionnels de la culture. Les représentations de l'identité kanak en Nouvelle-Calédonie (1975-2015). Montpellier, Université Paul-Valery Montpellier 3, thèse de doctorat.

\section{Guiart, Jean}

1956 Un siècle et demi de contacts culturels à Tanna, Nouvelles-Hébrides. Paris, Société des océanistes ("Publications de la Société des océanistes»).

Hassall, Graham

1991 "Church and State in Vanuatu 1945-1980: A "Pacific" Contest for Power", South Pacific Journal of Mission Studies 2 (2): 2-12.

2005 "The Bahá'í Faith in the Pacific», in Phyllis Herda, Michael Reilly \& David Hilliard, eds, Vision and Reality in Pacific Religion. Essays in Honour of Niel Gunson. Canberra,

Pandanus Books: 267-287.

Keesing, Roger M. \& Robert Tonkinson, eds 1982 Mankind 13 (4): Reinventing Traditional Culture. The Politics of Kastom in Island Melanesia. Sidney, Anthropological Society of New South Wales.

\section{Marc Tabani}


Kohler, Jean-Marie

1982 "L'islam en Nouvelle-Calédonie», L'Afrique et l'Asie modernes 135: 3-11.

\section{Lanternari,Vittorio}

1962 Les Mouvements religieux de liberté et de salut des peuples opprimés. Trad. de l'italien par Robert Paris. Paris, Maspero («Les Textes à l'appui»).

\section{Lindstrom, Lamont}

1993 Cargo Cult. Strange Stories of Desire from Melanesia and Beyond. Honolulu, University of Hawaii Press ("South Sea Books»).

2017 "Respek et autres mots-clés du PortVila urbain ", Journal de la Société des océanistes 144-145 (1) : 23-36. En ligne: https://journals.openedition.org/jso/7676

\section{Lini,Walter Hadye}

1980 Beyond Pandemonium.

From the New Hebrides to Vanuatu.

Wellington, Asia Pacific Books.

\section{McDougall, Debra}

2009 «Becoming Sinless: Converting to Islam in the Christian Solomon Islands ", American Anthropologist 111 (4) : 480-491.

\section{Marett, Robert Ranulph}

1928 "Foreword», in Francis Edgar Williams, Orokaiva Magic. Oxford, Clarendon Press: IX-XII.

Miles, William F. S.

1998 Bridging Mental Boundaries in a Postcolonial Microcosm. Identity and Development in Vanuatu. Honolulu, University of Hawaii Press.

Myers, Michael David

1984 Independens Long Vanuatu.

The Churches and Politics in a Melanesian

Nation. Auckland, University of Auckland, PhD.

\section{Newland, Lynda \& Terry M. Brown}

2015 «Introduction: Descent from Israel and Jewish Identities in the Pacific, Past and Present», Oceania 85 (3) : 251-255.

\section{Otto, Ton \& Poul Pedersen}

2005 «Disentangling Traditions: Culture, Agency and Power ", in Ton Otto \& Poul Pedersen, eds, Tradition and Agency. Tracing Cultural Continuity and Invention. Aarhus, Aarhus University Press : 11-50.

Possamai, Adam et al.

2016 "Shari' a and Everyday Life in Sydney", Australian Geographer 47 (3) : 341-354.

Robbins, Joel

2007 "Continuity Thinking and the

Problem of Christian Culture: Belief, Time, and the Anthropology of Christianity", Current Anthropology 48 (1) : 5-38.

Slama, Martin

2015 «Papua as an Islamic Frontier: Preaching in "the Jungle" and the Multiplicity of Spatio-Temporal Hierarchisations ", in Martin Slama \& Jenny Munro, eds, From "Stone-Age" to "Real-Time». Canberra, Autralian National University Press: 243-270.

\section{Strong, Walter $M$.}

1923 "Introduction", in F. Williams, The Vailala Madness and the Destruction of Native Ceremonies in Gulf Division. Port Moresby, Edward George Baker, Government Printer: I-XIII.

Tabani, Marc

2002 Les Pouvoirs de la coutume à Vanuatu. Traditionalisme et édification nationale. Paris, L'Harmattan («Connaissance des Hommes»)

2008 Une pirogue pour le paradis. Le culte de John Frum à Tanna (Vanuatu). Paris, Éd. de la Msh.

2019 «Tannese Chiefs, State Structures, and Global Connections in Vanuatu ", The Contemporary Pacific 31 (1) : 65-103.

Timmer, Jaap

2015 «Heirs to Biblical Prophecy:

The All Peoples Prayer Assembly in Solomon Islands", Nova Religio. The Journal of Alternative and Emergent Religions 18 (4): 16-34. 
Vanuatu National Statistic Office (VNSO)

2009 National Population and Housing Census. Analytical Report. Port-Vila, Ministry of Finance and Economic Management.

Wittersheim, Éric

2006 Après l'indépendance. Le Vanuatu, une démocratie dans le Pacifique.

La Courneuve, Aux lieux d'être.
Zocca, Franco

2006 "Vanuatu", in Manfred Ernst, ed.,

Globalization and the Re-shaping

of Christianity in the Pacific Islands.

Suva, Pacific Theological College:

205-264.

\section{RÉSUMÉ/ABSTRACT}

Marc Tabani, L'islam des musulmans de Tanna (Vanuatu). - L'islam comme croyance religieuse, imaginaire culturel et monde social est longtemps resté ignoré des sociétés du Pacifique sud. Dans des pays densément peuplés de la Mélanésie, tels que la PapouasieNouvelle-Guinée, les îles Salomon ou Vanuatu, s'employant depuis leur indépendance à construire des identités nationales fermement ancrées dans le christianisme, l'introduction de l'islam nécessite d'être étudiée en tant que phénomène strictement postcolonial. Â Vanuatu, après plusieurs décennies de souveraineté, les autorités politiques et religieuses du pays ont néanmoins cherché à réaffirmer sur un plan national l'hégémonie chrétienne. Cependant, du fait de l'ampleur des conséquences culturelles de la mondialisation et de la sécularisation, les populations de Vanuatu traversent des bouleversements sociaux qui s'accompagnent d'une crise morale parmi les Églises historiques du pays, confrontées au succès croissant des Églises néo-évangéliques et pentecôtistes. La conversion à l'islam, dans un tel contexte, représente un phénomène religieux nouveau d'un intérêt particulier pour les spécialistes en sciences sociales, puisqu'il relance les débats sur les politiques culturelles et la question de l'identité. Ce qui est alors en jeu, c'est la capacité de l'islam à se conformer ou non aux expériences mélanésiennes passées en matière d'indigénisation des influences culturelles étrangères. L'objectif de cet article est d'analyser les raisons de cette adhésion à cette nouvelle offre dans un véritable «marché de la foi », dans certains contextes et notamment sur l'île de Tanna, et ce, malgré une profonde méfiance à l'égard de l'islam.
Marc Tabani, Islam of Tannese Muslims (Vanuatu). - As religious belief, cultural construct and social world, Islam has long remained unknown to many South Pacific societies. In densely populated Melanesian countries like Papua New Guinea, the Solomon Islands and Vanuatu, that have, since their independence, concentrated on constructing national identities firmly rooted in Christianity, the introduction of Islam needs be studied as a specifically postcolonial phenomenon. In Vanuatu, after several decades of sovereignty, the political and religious authorities of this country have nonetheless felt the need to reassert Christianity's hegemony at the national level. However, due to the magnitude of the cultural consequences of globalization and secularization, the people of Vanuatu are going through social upheavals which are coupled with a moral crisis among the traditional Christian denominations, confronted by the growing success of neo-Evangelical and Pentecostal churches. Converting to Islam, in such a context, is a religious innovation of special interest for social science specialists since, in Vanuatu, this study involves debates on the politics of culture and identity. What is at stake in this issue is the ability of Islam to conform, or not, to past Melanesian experiences in indigenizing foreign cultural influences. The aim of this article is to analyze the reasons for adhering to this new offer on the «religion market", in certain contexts, and in particular, on the island of Tanna, despite a deep mistrust of Islam. 